\title{
Weighted Inequality
}

\author{
Santosh Ghimire
}

Department of Engineering Science and Humanities, Central Campus, Pulchowk, IOE, TU, Lalitpur, Nepal

Corresponding Email: santoshghimire@ioe.edu.np

\begin{abstract}
In this paper, we define Ap weights, briefly discuss the theory of weighted inequalities and its application and importance in various fields. We then prove that for an Ap weight function $\mathrm{w}$ and for some $\geq \mathbf{1}$, the function, $\min (\mathrm{w}, \mathrm{k})$ is an Ap weight function. Finally we establish the weighted inequality for $\min (w, k)$.
\end{abstract}

\section{Introduction}

The theory of weights has applications in variety of fields such as vector-valued inequalities, extrapolation theory and estimates for certain class of non linear differential equation. Furthermore, they are widely used in the study of boundary value problems for Laplace's equation in Lipschitz domains. In 1970, Muckenhoupt characterized positive functions $\mathrm{w}$ for which the Hardy-Littlewood maximal operator M maps Lp(Rn, w(x)dx) to itself. Muckenhoupt's characterization actually gave the better understanding of theory of weighted inequalities which then led to the introduction of Ap class and consequently the development of weighted inequalities. Weighted inequalities are used widely in harmonic analysis. For more about the theory of weights, refer [1].

In order to establish the weighted inequality, some definitions are in order.

Definition: The uncentered Hardy-Littlewood maximal operators on $\mathrm{R}^{\mathrm{n}}$ over balls $\mathrm{B}$ is defined as

$$
M(f)(x)=\sup _{x \in B} \operatorname{Avg}|f|=\sup _{x \in B} \frac{1}{|B|} \int_{B}|f(y)| d y .
$$

Similarly the uncentered Hardy-Littlewood maximal operators on $\mathrm{R}^{\mathrm{n}}$ over cubes $\mathrm{Q}$ is defined as

$$
M_{c}(f)(x)=\sup _{x \in Q} \operatorname{Avg}|f|=\sup _{x \in Q} \frac{1}{|Q|} \int_{Q}|f(y)| d y .
$$

In each of the definition above, the suprema are taken over all balls $\mathrm{B}$ and cubes $\mathrm{Q}$ containing the point x. H-L maximal functions are widely used in Harmonic Analysis. For the details about the H-L maximal operators, see [2].

Definition: A locally integrable function on $\mathrm{R}^{\mathrm{n}}$ that takes values in the interval $(0, \infty)$ almost everywhere is called a weight. So by definition a weight function can be zero or infinity only on a set whose Lebesgue measure is zero. 
We use the notation $w(E)=\int_{E} w(x) d x$ to denote the w-measure of the set $\mathrm{E}$ and we reserve the notation $\mathrm{L}^{\mathrm{p}}\left(\mathrm{R}^{\mathrm{n}}, \mathrm{w}\right)$ or $\mathrm{L}^{\mathrm{p}}(\mathrm{w})$ for the weighted $\mathrm{L}^{\mathrm{p}}$ spaces. We note that $\mathrm{w}(\mathrm{E})<\infty$ for all sets $\mathrm{E}$ contained in some ball since the weights are locally integrable functions.

Definition: A function $\mathrm{w}(\mathrm{x}) \geq 0$ is called an $\mathrm{A}_{1}$ weight if there is a constant $\mathrm{C}_{1}>0$ such that

$$
M(w)(x) \leq C_{1} w(x)
$$

where, $M(w)$ is uncentered Hardy-Littlewood Maximal function given by

$$
M(w)(x)=\sup _{x \in B} \frac{1}{|B|} \int_{B} w(t) d t .
$$

If $\mathrm{w}$ is an $\mathrm{A}_{1}$ weight, then the quantity (which is finite) given by

$$
[w]_{A_{1}}=\sup _{Q \text { cubes inR }}\left(\frac{1}{|Q|} \int_{Q}|w(t)| d t\right)\left\|w^{-1}\right\|_{L^{\infty}(Q)}
$$

is called the $A_{1}$ characteristic constant of $w$.

Definition: Let $1<\mathrm{p}<\infty$. A weight $\mathrm{w}$ is said to be of class $\mathrm{A}_{\mathrm{p}}$ if $[\boldsymbol{w}]_{A_{p}}$ is finite where $[\boldsymbol{w}]_{A_{p}}$ is defined as

$$
[w]_{A_{p}}=\sup _{Q \text { cubes in } \mathrm{R}^{\mathrm{n}}}\left(\frac{1}{|Q|} \int_{Q}|w(x)| d x\right)\left(\frac{1}{|Q|} \int_{Q} \mid w(x)^{\frac{-1}{p-1}} d x\right)^{p-1} .
$$

We remark that in the above definition of $A_{1}$ and $A_{p}$ one can also use set of all balls in $R^{n}$ instead of all cubes in $\mathrm{R}^{\mathrm{n}}$. Now we prove an weighted inequality. For this let $w$ is an $A_{p}$ weight function for some $1 \leq p<\infty$ and $k \geq 1$. We first show that $\min (\mathrm{w}, \mathrm{k})$ is in $\mathrm{A}_{\mathrm{p}}$ and satisfies the inequality

$$
[\min (w, k)]_{A_{p}} \leq c_{p}\left([w]_{A_{p}}+1\right)
$$

where $c_{p}=1$ when $p \leq 2$ and $c_{p}=2^{p-2}$ when $p>2$.

We first note that

$$
\min (w, k)^{\frac{-1}{\mathrm{p}-1}} \leq w^{\frac{-1}{\mathrm{p}-1}}+k^{\frac{-1}{\mathrm{p}-1}}
$$

implies:

$$
\frac{1}{|Q|} \int_{Q} \min (w, k)^{\frac{-1}{\mathrm{p}^{-1}}} d x \leq \frac{1}{|Q|} \int_{Q} w^{\frac{-1}{\mathrm{p}-1}} d x+k^{\frac{-1}{\mathrm{p}-1}}
$$

Thus,

$$
\left(\frac{1}{|Q|} \int_{Q} \min (w, k)^{\frac{-1}{\mathrm{p}-1}} d x\right)^{\mathrm{p}-1} \leq\left(\frac{1}{|Q|} \int_{Q} w^{\frac{-1}{\mathrm{p}-1}} d x+k^{\frac{-1}{\mathrm{p}-1}}\right)^{\mathrm{p}-1}
$$

First, we have the following inequality $\forall p \in(0,1), a, b \geq 0$, 


$$
1=\frac{a}{a+b}+\frac{b}{a+b} \leq\left(\frac{a}{a+b}\right)^{p}+\left(\frac{b}{a+b}\right)^{p}
$$

or equivalently

$$
(a+b)^{p} \leq a^{p}+b^{p} \forall p \in(0,1), a, b \geq 0
$$

Secondly, by Jensen's inequality, we have, for $\forall a, b \geq 0, p \geq 1$

$$
\left(\frac{a+b}{2}\right)^{p} \leq \frac{a^{p}+b^{p}}{2}
$$

Then,

$$
(a+b)^{p} \leq 2^{p-1}\left(a^{p}+b^{p}\right) \forall a, b \geq 0, p \geq 1
$$

Let us consider the case $1 \leq p \leq 2$. Using (1) we have

$$
\left(\frac{1}{|Q|} \int_{Q} w^{\frac{-1}{\mathrm{p}-1}} d x+k^{\frac{-1}{\mathrm{p}-1}}\right)^{\mathrm{p}-1} \leq\left(\frac{1}{|Q|} \int_{Q} w^{\frac{-1}{\mathrm{p}-1}} d x\right)^{\mathrm{p}-1}+\mathrm{k}^{-1}
$$

Moreover,

$$
\frac{1}{|Q|} \int_{Q} \min (w, k) d x \leq \min \left\{k, \frac{1}{|Q|} \int_{Q} w d x\right\}
$$

Hence,

$$
\begin{aligned}
& \left(\frac{1}{|Q|} \int_{Q} \min (w, k) d x\right)\left(\frac{1}{|Q|} \int_{Q} \min (w, k)^{\frac{-1}{\mathrm{p}-1}} d x\right)^{p-1} \\
\leq & \min \left\{k, \frac{1}{|Q|} \int_{Q} w d x\right\}\left(\left(\frac{1}{|Q|} \int_{Q} w^{\frac{-1}{\mathrm{p}-1}} d x+\right)^{p-1}+k^{-1}\right) \\
\leq & {\left[\left(\frac{1}{|Q|} \int_{Q} w d x\right)\left(\frac{1}{|Q|} \int_{Q} w^{\frac{-1}{\mathrm{p}-1}} d x+\right)^{p-1}+k k^{-1}\right] \leq[w]_{A_{p}}+1 . }
\end{aligned}
$$

This implies:

$$
[\min (w, k)]_{A_{p}} \leq[w]_{A_{p}}+1
$$

For $p \geq 2$, using (2), we have

$$
\left(\frac{1}{|Q|} \int_{Q} w^{\frac{-1}{\mathrm{p}-1}} d x+k^{\frac{-1}{\mathrm{p}-1}}\right)^{\mathrm{p}-1} \leq 2^{\mathrm{p}-2}\left[\left(\frac{1}{|Q|} \int_{Q} w^{\frac{-1}{\mathrm{p}-1}} d x+\right)^{p-1}+k^{-1}\right]
$$

Hence,

$$
\left(\frac{1}{|Q|} \int_{Q} \min (w, k)^{\frac{-1}{\mathrm{p}-1}} d x\right)^{p-1} \leq\left(\frac{1}{|Q|} \int_{Q} w^{\frac{-1}{\mathrm{p}-1}} d x+k^{\frac{-1}{\mathrm{p}-1}}\right)^{\mathrm{p}-1}
$$




$$
\leq 2^{\mathrm{p}-2}\left[\left(\frac{1}{|Q|} \int_{Q} w^{\frac{-1}{\mathrm{p}-1}} d x+\right)^{p-1}+k^{\frac{-1}{\mathrm{p}-1}}\right]
$$

Therefore,

$$
\begin{gathered}
\left(\frac{1}{|Q|} \int_{Q} \min (w, k) d x\right)\left(\frac{1}{|Q|} \int_{Q} \min (w, k)^{\frac{-1}{\mathrm{p}-1}} d x\right)^{p-1} \\
\leq \min \left\{k, \frac{1}{|Q|} \int_{Q} w d x\right\} 2^{\mathrm{p}-2}\left(\left(\frac{1}{|Q|} \int_{Q} w^{\frac{-1}{\mathrm{p}-1}} d x+\right)^{p-1}+k^{-1}\right) \\
\leq 2^{\mathrm{p}-2}\left[\left(\frac{1}{|Q|} \int_{Q} w d x\right)\left(\frac{1}{|Q|} \int_{Q} w^{\frac{-1}{\mathrm{p}-1}} d x+\right)^{p-1}+k k^{-1}\right] \\
\leq 2^{\mathrm{p}-2}\left([w]_{A_{p}}+1\right)
\end{gathered}
$$

This implies,

$$
[\min (w, k)]_{A_{p}} \leq 2^{\mathrm{p}-2}\left([w]_{A_{p}}+1\right)
$$

\section{References}

[1] Loukas Grafakos, Modern Fourier Analysis, Second Edition, Springer 2009.

[2] R. Ba nelos and C. N. Moore, Probabilistic Behavior of Harmonic Functions, Birkhauser Verlag, 1991. 